\title{
RDUS
}

Revue de DROIT UNIVERSITÉ DE SHERBROOKE

Tiłre : $\quad$ L'AVANCE ET LE DÉCOUVERT DE BANQUE EN COMPTE COURANT

Auteur(s): $\quad$ Marc DUVAL

Revue: $\quad$ RDUS, 1999-2000, volume 30, numéro 1

Pages: $\quad$ 183-204

ISSN : $\quad 0317-9656$

Éditeur : Université de Sherbrooke. Faculté de droit.

URI : $\quad$ http://hdl.handle.net/11143/12356

DOI : https://doi.org/10.17118/11143/12356 
Page vide laissée intentionnellement. 


\title{
COMMENTAIRE
}

\section{L'AVANCE ET LE DÉCOUVERT DE BANQUE EN COMPTE COURANT}

\author{
par Marc DUVAL*
}

Le compte courant est une convention par laquelle deux parties en relations d'affaires suivies conviennent d'affecter l'ensemble de leurs créances à un compte unique et de renoncer à l'individualité de chaque créance pour ne reconnaître que le solde du compte, constitué des articles de crédit et de débit, exigible en tout temps. Puisque le compte courant enregistre les créances des diverses opérations entre les parties au compte, il est donc important que nous soyons en mesure de déterminer le moment précis où une créance entre en compte. L'avance et le découvert de banque nous permettront d'illustrer nos propos.

A current account is an agreement by which two parties in an ongoing business relationship, agree to assign the whole of their debts to a single account and to waive the individual nature of each debt and recognize only the balance of the account, made up of the debit and credit balances payable at all times. Since a current account records debts deriving from the various transactions between the two titularies of the account, it is therefore important to be able to determine the precise moment a debt actually enters the account. This is indicated through records of bank advances and overdrafts.

*. M. Fisc. Direction générale de la législation, ministère du Revenu du Québec. Les opinions exprimées dans cet article n'engagent que l'auteur. L'auteur remercie Gaétan Lépine de la Direction générale de la législation pour la vérification des écritures comptables. Des remerciements particuliers vont à Pierre Gagné également de la Direction générale de la législation pour les précieux commentaires qu'il a formulés à l'égard de ce texte ainsi que pour les nombreuses heures passées à discuter des concepts qui y sont énoncés. 


\section{SOMMAIRE}

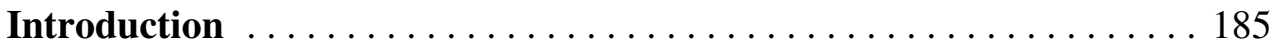

Distinctions et similitudes : l'avance et le découvert $\ldots \ldots \ldots 6$

La remise en compte courant $\ldots \ldots \ldots \ldots \ldots \ldots \ldots \ldots \ldots \ldots \ldots \ldots$

L'avance en compte courant $\ldots \ldots \ldots \ldots \ldots \ldots \ldots \ldots \ldots \ldots \ldots \ldots$

Le découvert en compte courant $\ldots \ldots \ldots \ldots \ldots \ldots$. . . . . . 198

L'effet novatoire et les ouvertures de crédit au Québec . . . . . . . . . 200

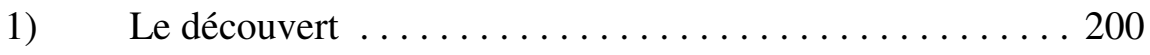

2) La protection contre le découvert : l'avance de fonds . . . . 202

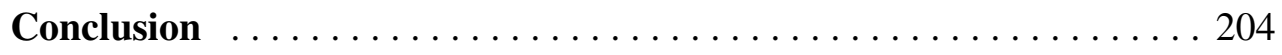




\section{Introduction}

Le compte courant est une convention par laquelle deux parties en relations d'affaires suivies conviennent d'affecter l'ensemble de leurs créances à un compte unique et de renoncer à l'individualité de chaque créance pour ne reconnaître que le solde du compte, constitué des articles de crédit et de débit, exigible en tout temps. Deux caractéristiques ressortent particulièrement de la convention de compte courant : l'absence d'imputation de paiements et l'effet novatoire du compte. ${ }^{1}$ Toutefois, l'effet novatoire est probablement l'élément essentiel de la convention de compte courant.

Le compte courant enregistre les créances résultant des diverses opérations effectuées entre les parties au compte. Il devient important d'établir le moment où une créance entre en compte courant car elle subit l'effet novatoire du compte et est remplacée par le solde du compte. En outre, par l'effet novatoire du compte, les garanties afférentes à la créance entrée en compte sont éteintes. Ainsi, la créance en remboursement de fonds prêtés inscrite en compte fera non seulement disparaître le prêt mais également les sûretés qui pourraient avoir été consenties à cet égard. Or, il n'est pas toujours facile de déterminer à quel moment une créance entre en compte courant. L'avance et le découvert de banque en compte courant nous permettront de saisir les aspects de l'inscription d'une créance en compte courant.

Nous distinguerons dans un premier temps l'avance du découvert, bien que les deux notions révèlent certaines similitudes. D'autre part, l'effet novatoire étant relié à la notion de remise en compte courant, cette notion sera analysée afin de nous permettre de qualifier l'opération à l'origine de la créance inscrite en compte courant. Par la suite, nous déterminerons à quel moment les créances relatives à l'avance et au découvert sont inscrites en compte courant. Enfin, nous examinerons, dans le cadre de l'effet novatoire du compte courant, deux types de clause que nous retrouvons dans des ouvertures de crédit utilisées au Québec et

1. Nous suggérons au lecteur de lire notre article précédent sur le compte courant avant d'entreprendre la lecture du présent texte s'il ne possède aucune connaissance concernant le compte courant; voir M. Duval, «Le compte courant en droit commercial» (1997) 57 R. du B. 453. 

protection contre le découvert.

Avant de débuter l'étude de notre sujet, il est important de noter que le lecteur pourrait s'interroger sur les motifs pour lesquels nous référerons presque exclusivement à la doctrine française dans le cadre de notre exposé. Ceci s'explique par l'absence de doctrine québécoise sur l'effet novatoire en compte courant. Puisque la Cour d'appel du Québec a référé à la doctrine française pour définir le compte courant, nous sommes également d'avis qu'il est pertinent d'y référer. $^{2}$

\section{Distinctions et similitudes : l'avance et le découvert}

Le client d'une institution financière peut obtenir des fonds notamment par l'avance ou le découvert dans le cadre d'une ouverture de crédit. L'ouverture de crédit est «une promesse unilatérale du banquier de mettre sous certaines conditions de temps et de taux à la disposition de son client des moyens de paiement sous une ou plusieurs formes.» ${ }^{3}$ Elle constitue une promesse de crédit. Généralement, la convention d'ouverture de crédit se greffe à une convention de compte courant.

2. Voir les arrêts Cayouette ltée c. Billet, [1962] B.R. 433. et Hamel c. Lauzon, [1978] C.A. 348. Voir également Banque de Montréal c. Québec (P.G.), [1979] R.C.S. 565 où la Cour Suprême du Canada réfère aux ouvrages de Rodière, de Rives-Lange, de Planiol, d'Aubry et Rau et de Mazeaud à des fins de discussion sur la nature juridique du contrat bancaire. D'autre part, nous référons le lecteur à un texte du professeur Pierre-Gabriel Jobin sur l'influence de la doctrine française à l'égard du droit civil québécois. P.-G. Jobin, Droit québécois et droit français, Cowansville (Qc.), Yvon Blais, 1993 à la p. 91 et s.

3. C. Gavalda et J. Stouflet, Droit bancaire, Paris, Litec, 1994 à la p. 219. François Grua y voit une promesse «sui generis» dont l'objet est le crédit à consentir; F. Grua, Contrats bancaires, t. 1, Paris, Economica, 1990, p. 219. Voir également J.-L. Rives-Lange et M. ContamineRaynaud, Droit bancaire, Paris, Dalloz, 1995 à la p. 445 et s.; A. Dieryck, Les ouvertures de crédit, Paris, Librairie Générale de Droit, 1945 à la p. 417. Il est pertinent de citer des auteurs français sur ce sujet puisque Pierre-Paul Côté s'exprimant sur l'ouverture de crédit mentionne que « [1] droit français, on doit l'avouer, a été plus fertile dans la recherche de son caractère juridique.» À cet égard, il cite différentes définitions d'auteurs français sur l'ouverture de crédit et sur son caractère juridique et il souligne qu'«il ne s'agit pas nécessairement d'une promesse de prêt car le crédit peut être réalisé sous une autre forme que le prêt.» P.-P. Côté, «Considérations sur le prêt commercial et la convention de prêt » (1987) 28 C. de D. 864. 
Précisons que l'avance de fonds constitue un contrat de prêt d'argent. ${ }^{4} \mathrm{Le}$ prêt « peut se traduire en compte soit par une écriture au crédit soit par le fait que le banquier laisse le compte devenir débiteur. ${ }^{5}$ Bien que dans le cadre du découvert bancaire, la banque avance des fonds, nous distinguerons l'avance du découvert. ${ }^{6}$ Lorsqu'une écriture au crédit est faite par la banque au compte du client, nous qualifierons l'opération d'avance tandis que lorsque la banque laisse le compte devenir débiteur, il s'agira d'un découvert. ${ }^{7}$ Nous sommes d'avis que cette distinction traduit bien la réalité de ces opérations, bien que tant le découvert que l'avance constituent un prêt d'argent.

Toutefois, en compte courant, le découvert doit avoir été qualifié de prêt par les parties au compte afin que l'opération à l'origine de la créance soit qualifiée de prêt. À défaut d'une telle qualification, l'opération à l'origine de la créance qui résulte du transfert de numéraire ne peut être qualifiée de prêt comme nous le constaterons. Puisque lors d'une ouverture de crédit entre une banque et son client, le découvert est généralement qualifié de prêt, nous référerons à la notion de prêt, contrat nommé au Code civil du Québec, pour effectuer notre démonstration tant à l'égard de l'avance que du découvert.

4. Rives-Lange et Contamine-Raynaud s'expriment comme suit à l'égard du prêt : «Les prêts à court terme se présentent sous des modalités techniques très variées : avances, découverts, crédit de courrier, crédit de campagne, etc. Au point de vue juridique, ces différentes opérations se ramènent à une notion unique, celle du prêt; il n'y a diversité juridique qu'en ce qui concerne les sûretés consenties pour garantir le prêt.»

J.-L. Rives-Lange et M. Contamine-Raynaud, ibid. à la p. 460. Sur la notion d'avances de fonds, voir également G. Ripert et R. Roblot, Traité de droit commercial, $15^{\mathrm{e}}$ éd. par P. Delebecque et M. Germain, Paris, Librairie Générale de Droit et de Jurisprudence, 1996 à la p. 347; C. Gavalda et J. Stoufflet, ibid. à la p. 215; J. Escarra, Cours de droit commercial, Paris, Sirey, 1952 à la p. 964; J. Hamel, G. Lagarde et A. Jauffret, Traité de droit commercial, t. 2, Paris, Librairie Dalloz, 1967 à la p. 816; Marcelon Inc. c. M.R.N., [1991] R.D.F.Q. 7.

5. J.-L. Rives-Lange et M. Contamine-Raynaud, ibid. à la p. 461. Voir également G. Ripert et R. Roblot, ibid. à la p. 464.

6. J.-L. Rives-Lange et M. Contamine-Raynaud distinguent également ces deux notions : supra note 4.

7. La notion d'avance telle que nous l'entendons est celle-ci : «les avances sur créances [...]; les avances sur effets à encaisser; les avances sur marchandises; les avances sur recettes (films); les avances sur marchés; enfin les avances sur titres.» C. Gavalda et J. Stoufflet, supra note 3 à la p. 215. D'autre part, les concepts développés dans ce texte à l'égard de l'avance en compte courant peuvent être utilisés lorsqu'un prêt est octroyé par la banque au client et que la remise des fonds a lieu par une inscription au crédit du compte courant. 
Avant de commencer l'étude de l'avance en compte courant, nous nous permettons de faire quelques commentaires sur la notion de remise en compte courant.

\section{La remise en compte courant}

Le terme «remise» désigne la créance qui entre en compte. ${ }^{8}$ Nous avons mentionné dans un article précédent qu'une créance qui entre en compte courant doit être certaine, liquide et exigible conformément à la doctrine prépondérante. ${ }^{9}$ Or, l'étude de l'avance en compte courant nous permet d'affirmer que le caractère d'exigibilité de la créance n'est pas une condition pour entrer en compte courant. D'ailleurs, nous constatons que certains auteurs mentionnent que la créance doit être certaine et liquide et n'ajoutent pas le caractère d'exigibilité. ${ }^{10}$

L'avance en compte courant provient généralement d'une ouverture de crédit. Lors d'une avance de fonds, l'inscription de la créance au compte courant par une écriture au crédit matérialise la mise à la disposition des fonds au client par l'institution financière qui a consenti l'ouverture de crédit. Cette créance estelle exigible? L'ouverture de crédit prévoit que l'institution financière doit consentir le crédit mais certaines clauses de cette convention permettent généralement à cette dernière de se désister lors de l'avènement de certaines conditions, d'où le caractère douteux de l'exigibilité du crédit par le bénéficiaire. ${ }^{11}$

8. $\quad$ M. Duval, supra note 1 à la p. 466.

9. Ibid. à la p. 467. Voir notamment Encyclopédie juridique Dalloz : répertoire de droit commercial, $2^{\mathrm{e}}$ ed., «compte courant» par J. Stoufflet, au $\mathrm{n}^{\circ}$ 70; J.-L. Rives-Lange et M. Contamine-Raynaud, supra note 3 à la p. 237; M.-T. Calais-Auloy, Jurisclasseur, Droit commercial, compte-courant, paragraphe 53 et 76 .

10. G. Ripert et R. Roblot, supra note 4 à la p. 417. Marie-Thérèse Rives-Lange mentionne que «toute créance peut constituer une remise à la seule condition qu'elle appartienne au cadre de la convention initiale.» M.-T. Rives-Lange, Le compte courant en droit français, Sirey, Paris, 1969 à la p. 90. Le caractère consensuel du compte explique, selon nous, la possibilité qu'une créance non exigible puisse constituer une remise car le compte courant est la manifestation de la volonté des parties de régler leurs créances dans le cadre du compte.

11. François Grua mentionne que «l'ouverture de crédit a une force obligatoire limitée. En principe, cette obligation de faire qu'elle engendre ne commande pas que le banquier défère à tout appel de fonds du bénéficiaire, serait-ce dans la limite du montant prévu. Il conserve un certain pouvoir de sélection qui tient à deux raisons. [...] Dans ces conditions, la liberté du banquier de ne pas contracter vient singulièrement limiter la force obligatoire de la 
D'autre part, si l'opération à l'origine de l'inscription au crédit par l'institution financière était une promesse de prêt, la créance afférente à celle-ci n'est pas exigible puisque l'article 2316 de Code civil du Québec prévoit que la promesse de prêter ne confère au bénéficiaire de la promesse, à défaut par le promettant de l'exécuter, que le droit de réclamer des dommages-intérêts. ${ }^{12}$

Que peut-on conclure? Dans le cadre d'une avance en compte courant, une créance est inscrite en compte et ce montant au crédit du compte matérialise une créance provenant d'une ouverture de crédit. Cette créance, malgré son caractère d'inexigibilité, peut être inscrite en compte courant. ${ }^{13}$ La situation est similaire dans le cadre d'une promesse de prêt où les fonds sont mis à la disposition de l'emprunteur par l'inscription au compte d'une écriture au crédit qui matérialise la créance de l'emprunteur vis-à-vis l'institution financière à l'égard de la promesse de prêt; le prêt est formé par la mise à la disposition des fonds au

promesse; il n'a promis qu'en général, rien dans le particulier. Il y a seulement une réserve, sans laquelle la promesse serait purement potestative, mais qui est bien ténue : la liberté du banquier de refuser de répondre à un appel de crédit doit s'exercer dans la limite de l'abus de droit.» F. Grua, supra note 3 à la p. 230. Ripert et Roblot mentionnent que la «convention est unilatérale, en ce sens que le banquier est obligé de consentir le crédit, mais le client n'est pas obligé d'en user. Toutefois, quand elle est faite en compte courant, le client se trouve tenu de passer toutes ses opérations en compte». G. Ripert et R. Roblot, supra note 4 à la p. 459.

12. Dans le cadre d'un prêt accordé par une institution financière où les fonds ont été mis à la disposition du client par une inscription au crédit du compte courant, Jean-Louis RivesLange s'exprime comme suit : «Le contrat de prêt - il vaudrait mieux dire la promesse de prêt - obligeait la banque à mettre les fonds prêtés à la disposition de l'emprunteur; voilà l'obligation de la banque et la créance corrélative de l'emprunteur qui a été porté au crédit du compte courant ! Cette créance a subi l'effet novatoire du compte courant ». J.-L. RivesLange, Chronique de jurisprudence bancaire, La Revue Banque, septembre 1987, p. 852. Jean-Louis Rives-Lange ne semble pas s'être interrogé sur l'exigibilité de cette créance. Dans son volume sur le droit bancaire, il mentionne que pour que la créance subisse les effets du compte courant, elle doit être « certaine, liquide et exigible»; J.-L. Rives-Lange et M. Contamine-Raynaud, supra note 3 à la p. 237. Or, Jean-Régis Mirbeau-Gauvin dans le Répertoire civil Dalloz mentionne, référant à un arrêt de la Cour de cassation, que «[1]e bénéficiaire de la promesse de prêt n'a droit qu'à des dommages-intérêts, en cas d'inexécution, et non à l'exécution en nature». (Voir Encyclopédie juridique Dalloz : répertoire de droit civil, «Prêt» par J.-R. Mirbeau-Gauvin au n ${ }^{\circ} 264$ ). Nous pouvons donc, à l'instar du droit civil québécois, qualifier d'inexigible la créance du bénéficiaire de la promesse de prêt.

13. Voir G. Ripert et R. Roblot, supra note 4 à la p. 417. 

permet d'en conserver l'autonomie et de préserver la nature de l'opération (du contrat) qui est à l'origine de la créance entrée en compte lors d'une écriture au crédit par l'institution financière. ${ }^{15}$

La créance qui entre en compte courant résulte des diverses opérations faites entre les deux parties au compte. Dans l'étude du compte courant, cette notion d'opération est moins importante puisque c'est la créance qui entre en compte courant et non l'opération à l'origine de cette créance. Toutefois, cette notion d'opération permet de déterminer, lorsqu'une écriture au crédit du compte est effectuée par l'institution financière, quelle créance subit l'effet novatoire du compte courant.

14. Les deux parties dans le cadre d'un prêt peuvent prévoir que la remise peut s'effectuer par une écriture au crédit du compte courant. Il s'agit de la tradition feinte : «La tradition nécessaire à la formation du contrat peut résulter du seul consentement des parties, sans qu'il soit nécessaire d'opérer effectivement une livraison matérielle. La tradition feinte - fictive , connu sous le nom de tradition brevi manu, suffit pour opérer le prêt d'une somme d'argent (Cass. req. 29 mars 1887, DP 89.1.159, note 1-2). Elle peut se réaliser, par exemple, sous forme d'écritures comptables ou bancaires (M. Planiol et G. Ripert, Traité pratique de droit civil français, t. 11, $2^{\mathrm{e}}$ éd., Paris, Librairie générale de droit et de jurisprudence, 1954, au $\mathrm{n}^{\mathrm{o}}$ 1141).» Encyclopédie juridique Dalloz : répertoire de droit civil, «Prêt» par J.-R. MirbeauGauvin au ${ }^{\circ} 257$.

Jean-Louis Rives-Lange mentionne que «[p]ar l'acte de prêt, la banque a souscrit l'obligation de mettre les fonds à la disposition de son client emprunteur». J.-L. Rives-Lange, supra note 12 à la p. 853. Au même effet, voir Michel Cabrillac et Bernard Teyssié, infra note 21. Nous comprenons que la tradition feinte équivaut à une mise à la disposition des fonds puisqu'elle se traduit en compte courant par l'inscription d'une créance en faveur du client.

15. L'autre possibilité serait de conclure à l'autonomie du contrat de prêt par rapport à la convention du compte courant. Celle-ci a été soutenue en France par la Cour de cassation dans une décision isolée et sévèrement critiquée par J.-L. Rives-Langes; voir J.-L. RivesLanges, supra note 12; au même effet, voir M. Cabrillac et B. Teyssié, «Chroniques de la Législation et de Jurisprudence française» (1987) 40 R.T.D. civ. et com. 851. Selon la théorie énoncée par la Cour de cassation, les contractants au compte seraient d'accord pour déroger aux conséquences (l'effet novatoire) de l'inscription du montant du prêt au compte courant. Quant à la théorie du compte courant telle qu'exposée, elle permet de préserver l'autonomie des opérations à l'origine de la créance entrée en compte, ceci dans le cadre d'une écriture au crédit par l'institution financière (le prêt subsiste) tandis qu'une écriture au débit par cette dernière dans le cadre d'un prêt viserait la créance en remboursement des fonds prêtés, d'où la disparition du prêt par l'effet novatoire du compte. Enfin, nous soulignons que la théorie du compte courant étant d'origine doctrinale et jurisprudentielle, il est légitime que ce concept évolue et qu'il s'adapte aux besoins commerciaux. 
À titre d'exemple, supposons que monsieur $\mathrm{A}$ et la banque $\mathrm{X}$ sont en compte courant. Si monsieur A va faire un «dépôt» de 100 \$à son compte à la banque $\mathrm{X}$, l'opération juridique qui naît du dépôt pourrait être un prêt (la créance en remboursement des fonds prêtés est inscrite au compte), dans la mesure où ce contrat respecte les conditions énoncées au Code civil du Québec et où les parties ont consenti au prêt. Toutefois, rien n'interdît en compte courant que nous soyons en présence d'un transfert de numéraire sans qu'une opération juridique soit à l'origine du dit transfert. Ainsi, lors d'un transfert de numéraire du remettant au récepteur, le compte courant enregistre la créance résultant de ce transfert; du fait que le remettant transfère de l'argent au récepteur, une créance naît pour matérialiser le transfert, que le solde du compte soit débiteur ou créditeur. ${ }^{16}$ Nous qualifions cette opération de matérielle lorsque l'opération à l'origine de la créance perd tout caractère propre par opposition à une opération juridique tel un prêt. D'ailleurs, rien ne s'oppose à ce que le «dépôt» de $100 \$$ de monsieur A à la banque $B$ soit plutôt une opération matérielle que juridique, c'est-à-dire que du

16. Vasseur et Marin définissent les termes «remettant» et « récepteur» comme suit : «Le remettant est celui qui apparaît comme créancier de la créance passée en compte; c'est en pratique celui qui remet à l'autre la chose ou la valeur dont le transfert fait naître la créance. Le récepteur est au contraire le débiteur de la créance; c'est celui qui reçoit du remettant la chose ou la valeur dont il doit la restitution ou le prix.» M. Vasseur et X. Marin, Les comptes en banque, t. 1, Sirey, Paris, 1966 à la p. 389. 
«Du fait de leur entrée en compte, les remises perdent leur caractère de paiements. En l'absence de compte, une remise constituerait, soit un paiement fait par le remettant au récepteur, soit une avance si le remettant n'était pas débiteur du récepteur. La remise faite par le banquier au profit du client pourrait avoir le caractère d'une avance; c'est ce qui se produirait dans le cas d'un compte de dépôt d'espèces. Faite en compte courant, la remise n'est plus une avance. Il reste simplement qu'une somme est entrée dans le compte, somme dont le récepteur a acquis la propriété.» ${ }^{18}$

17. Les commentaires qui précèdent nous amènent au développement théorique suivant sur la nature du compte courant. Bien que la créance en remboursement des fonds prêtés, qui tire son origine du prêt entre les deux parties au compte (le remettant a remis de l'argent au récepteur dans le cadre d'un prêt et de cette transmission résulte une créance en remboursement des fonds prêtés), soit inscrite immédiatement au compte après le transfert de numéraire, nous favorisons de façon générale dans le cadre d'un transfert de numéraire la notion d'opération matérielle. Effectivement, dans le compte courant, les deux parties enregistrent dans un compte les créances résultant des diverses opérations intervenues entre elles dans le cadre d'une relation de créancier-débiteur et non dans un cadre plus spécifique de prêteur-emprunteur. En outre, cette notion est plus conforme aux effets juridiques du compte. En effet «[c]haque élément du compte courant perd sa nature particulière, son individualité, pour devenir un simple article de débit ou de crédit et concourir à la formation du solde qui constituera une créance ou une dette sui generis». H. Terrel et H. Lajeunesse, Traité des opérations commerciales de banque, Paris, Masson et Cie Éditeurs, 1921 à la p. 42. D'autre part, la notion d'opération matérielle à l'origine de la créance entrée en compte est plus conforme au fonctionnement du compte courant puisque lorsque le solde du compte du client est débiteur de la banque et que le client remet un montant d'argent à cette dernière, l'opération à l'origine de la remise au compte n'est pas un paiement. Sur cette question de paiement, voir M. Duval, supra note 1 à la p. 467. Voir également la note 18.

18. Les italiques sont de l'auteur. J. Escarra, supra note 4 à la p. 940. A. Boistel faisait également la même observation en 1890 : «Au point de vue juridique, ces remises, s'il n'y avait pas de compte courant, pourraient se présenter dans deux conditions opposées : si elles sont faites par une partie qui n'est pas déjà débitrice de l'autre, elles constitueraient une avance, un prêt; si elles émanent de celui qui est déjà d'ailleurs débiteur, elles seraient un paiement. Dans le compte courant ces remises perdent tout caractère propre; qu'elles soient au fond des avances ou des paiements, elles figurent au même titre au compte ». A. Boistel, Cours de droit commercial, 4 éd., Paris, Ernest Thorin, 1890, p. 613.

Cette observation est reprise par Albert Dieryck (infra note 31). Quant à nous, nous suggérons que, dans le cadre de l'avance et du découvert, c'est l'opération à l'origine de la créance qui perd tout caractère propre et que la créance perd également tout caractère propre 
Cette description de la remise qu'exprime le professeur Escarra nous apparaît incomplète. En effet, il y a lieu de noter que la remise ne représente la conséquence d'une opération matérielle (c'est-à-dire l'opération à l'origine de la créance perd tout caractère propre dans le cadre d'un transfert de numéraire) que lorsque la créance inscrite au compte matérialise la dette résultant du transfert de numéraire du remettant au récepteur tel dans le cas du découvert, du «dépôt» d'espèces lorsque le remettant n'est pas débiteur du récepteur et du «paiement». Ainsi, il ne saurait être question que l'avance tel que nous l'avons distinguée du découvert soit une opération matérielle puisque les parties ont qualifié de prêt l'opération intervenue entre elles et plus particulièrement parce que le montant inscrit au crédit matérialise une dette de la banque à l'égard du client plutôt que la dette résultant du transfert de numéraire du remettant (la banque) au récepteur (le client). ${ }^{19}$ De ce qui précède, nous sommes d'avis que la remise ne peut résulter que d'une opération matérielle ou d'une opération juridique.

\section{L'avance en compte courant}

Rappelons que pour toute obligation existe une créance, créance qui peut être inscrite en compte courant si elle est certaine et liquide. ${ }^{20}$ Que se passe-t-il

puisqu'elle n'est que le corollaire d'une opération effectuée entre les parties au compte. Par ailleurs, il est important de noter que les créances résultant d'opérations juridiques peuvent être inscrites en compte. À cet égard, Ripert et Roblot mentionnent que « [1]a créance du remettant envers le récepteur peut tenir aux causes juridiques les plus variées : paiement d'un prix de vente, prêt d'argent, ouverture de crédit, intérêts de titres, etc. Cette cause de la créance est sans importance dans la théorie du compte courant, sauf sur le point de savoir si elle ne doit pas la faire exclure du compte. » G. Ripert et R. Roblot, supra note 4 à la p. 417.

19. Ripert et Roblot mentionnent qu' «[i]l y a beaucoup de confusion dans la doctrine entre la capacité de faire une remise et les effets propres au compte courant. Cette confusion peut être surtout observé dans l'étude de l'ouverture de crédit en compte courant. Si le crédit ouvert est porté au compte du client et en est parfois le premier article, c'est que le banquier a pris, par un contrat d'ouverture de crédit, l'obligation de consentir un prêt.» G. Ripert et R. Roblot, supra note 4 à la p. 417.

20. Qu'est-ce qu'une obligation? Le professeur Tancelin mentionne que «[1]e droit civil définit traditionnellement l'obligation comme un lien de droit entre deux personnes.» Les frères Mazeaud ajoutent que pour la personne qui doit effectuer une prestation, «[1]'obligation est un élément du passif de son patrimoine, une dette. Or, l'obligation présente nécessairement une face inverse. Pour la personne qui doit profiter de la prestation due, elle est un élément de l'actif de son patrimoine, une créance.» M. Tancelin, Des obligations : «ctes et responsabilités, Montréal, Wilson \& Lafleur, 1997, p. 1; Henri, Léon et J. Mazeaud, Leçons 
lorsqu'une avance est faite en compte courant? Comme mentionné précédemment, une avance de fonds en compte courant a généralement lieu dans le cadre d'une ouverture de crédit. La réalisation d'avances en compte courant est une façon pour la banque d'exécuter son obligation prévue à l'ouverture de crédit. Ainsi, dans le cadre d'une avance de fonds, il y a deux obligations ou deux créances. La première obligation résulte de l'ouverture de crédit. Par le contrat d'ouverture de crédit, la banque a l'obligation de remettre l'argent à l'emprunteur; elle a une dette envers l'emprunteur. Si nous examinons la situation du côté du client, nous constatons qu'il a une créance envers la banque. D'autre part, nous avons mentionné que l'avance de fonds est un contrat de prêt d'argent. Le prêt d'argent est un simple prêt défini par l'article 2314 du Code civil du Québec comme étant un « contrat par lequel le prêteur remet une certaine quantité d'argent à l'emprunteur [...] qui s'oblige à lui en rendre autant, de même espèce et qualité, après un certain temps.» L'emprunteur doit donc remettre l'argent au prêteur : c'est l'obligation de l'emprunteur ou sa dette envers le prêteur et la créance du prêteur en remboursement des fonds prêtés. ${ }^{21}$ Nous constatons que nous

de droit civil, t. 2, Paris, Montchrestien, 1966 à la p. 5. En ce qui concerne le caractère d'exigibilité, voir nos propos précédents sur le sujet.

21. Supposons par ailleurs qu'il n'y ait aucune ouverture de crédit entre la banque et le client, mais qu'un contrat de prêt soit conclu entre eux et qu'une créance soit inscrite au crédit du client au compte courant. Puisque le montant du prêt n'est pas versé immédiatement (c'est-àdire qu'il y a un délai entre la signature du contrat et l'inscription de la créance au compte du client, qui peut avoir lieu dans la journée ou le lendemain du contrat), nous sommes d'avis que le contrat n'est pas un prêt mais plutôt une promesse de prêt puisque dans le cadre du contrat réel qu'est le prêt, la tradition de la chose doit avoir été effectuée pour que le contrat existe (pour une discussion sur le prêt, contrat réel, voir M. Deschamps, «Le prêt» dans $L a$ réforme du Code civil : obligations, contrats nommés, Sainte-Foy (Qc.), 1015). Or, dans ce cas, la tradition n'a lieu qu'ultérieurement à la signature du contrat, soit au moment de l'inscription au compte de la créance qui constate que la banque a rempli son obligation. Nous soumettons toutefois l'opinion de Michel Cabrillac et de Bernard Teyssié qui, dans le cadre d'un commentaire d'arrêt, mentionnent que « [p]ar l'inscription au crédit du compte de l'emprunteur, le banquier avait mis les fonds à sa disposition, exécutant par là l'obligation que la convention de prêt avait mis à sa charge. »; M. Cabrillac et B. Teyssié, supra à la note 15 de la p. 551. Ces deux auteurs sont donc d'avis que l'emprunteur possède une créance à l'égard du prêteur. Toutefois, il semble qu'en France on retienne que le contrat de prêt est un contrat réel comme au Québec bien que ce sujet ait fait l'objet de nombreuses discussions par la doctrine (Voir J.-R. Mirbeau-Gauvin, supra note 12 par. 225). Voir toutefois le commentaire de Didier Valette qui mentionne que «[s]'agissant d'un contrat réel, le contrat de prêt se forme théoriquement à la remise des fonds par le prêteur. Mais en raison de la pratique moderne des contrats de prêts, bon nombre d'auteurs hésitent à maintenir cette 
sommes en présence de deux créances dans le cadre de l'avance de fonds; chacune de ces créances pourrait faire l'objet d'une inscription en compte courant.

À des fins de compréhension de l'avance en compte courant dans le cas d'une ouverture de crédit, utilisons l'exemple suivant que nous désignerons l'exemple numéro 1. La banque $\mathrm{A}$ fait une avance de fonds à monsieur $\mathrm{B}$ pour un montant de 1000 \$. À cette fin, une écriture au montant de 1000 \$ est inscrite au crédit de monsieur $\mathrm{B}$ à son compte. Rappelons que toute créance qui subit l'effet novatoire du compte courant est remplacée par le solde du compte constitué des articles de débit ou de crédit. Il est important de rappeler à ce stade-ci que c'est la créance et non l'opération dont est issue la créance, c'est-à-dire le prêt, qui subit l'effet novatoire. ${ }^{22}$ De plus, il nous faut établir quelle créance est inscrite au compte en examinant les écritures de débit ou de crédit puisqu'il faut déterminer, lorsque le prêt est accordé dans le cadre d'une ouverture de crédit, quelle créance subit l'effet novatoire, soit celle relative à l'ouverture de crédit ou celle relative au remboursement des fonds prêtés. À ce propos, il convient de noter que «les écritures au crédit [dans les livres de la banque] représentent les créances du client sur la banque, et qu'à l'inverse, les écritures au débit représentent les créances de la banque sur le client ou, si l'on préfère une autre façon de s'exprimer, les dettes du client à l'égard de la banque. ${ }^{23}$ Comme l'écriture au débit matérialise une créance de la banque à l'encontre du client, c'est en examinant les écritures au débit que l'on pourra établir si la créance en remboursement des fonds prêtés a subi l'effet novatoire du compte courant.

Résumons la démarche à accomplir dans le cas de l'exemple numéro 1. Deux éléments doivent être examinés afin de déterminer si la créance en remboursement des fonds prêtés a subi l'effet novatoire du compte courant : 1. est-ce qu'il y a un montant inscrit au débit dans les livres de la banque : 2. ce montant

qualification de contrat réel. » (voir les références citées par cet auteur); Juris-classeur commercial : banque et crédit, Conventions de crédit par D. Valette au $\mathrm{n}^{\circ} 77$.

Quoi qu'il en soit, dans les deux situations, nous sommes d'avis que l'inscription au crédit du compte du client matérialise la créance du client à l'égard de la banque relativement à la remise de l'argent en vertu de la promesse de prêt ou du prêt en supposant que le prêt n'est pas un contrat réel.

22. J.-L. Rives-Lange, supra note 12 à la p. 853.

23. J.-L. Rives-Lange, supra note 12 à la p. 853. Voir également J.-L. Rives-Lange, Chronique de jurisprudence bancaire, La Revue Banque, mai 1986 à la p. 506. 
représente-t-il la créance visée, en l'occurrence, en remboursement des fonds prêtés. Notons que si c'était monsieur B qui effectuait le prêt à la banque, le premier élément à examiner serait les montants inscrits au crédit dans les livres de la banque, puisque seul un montant au crédit dans ce cas peut matérialiser la créance du client à l'égard de la banque ou, si l'on préfère, la dette de la banque à l'égard du client. Quant au deuxième élément, il demeure le même puisqu'il faut déterminer si le montant inscrit au compte représente la créance visée.

Revenons à notre exemple numéro 1. La banque a fait une avance de 1000 \$ à monsieur B, ce qui s'est traduit par une inscription de 1000 \$ au crédit de son compte. La banque avait l'obligation de remettre l'argent à monsieur B en vertu de l'ouverture de crédit qu'elle lui avait octroyée; c'était la dette de la banque envers monsieur B ou la créance de monsieur B envers la banque. Si nous examinons les écritures, nous constatons que l'écriture au crédit de la banque représente une dette de 1000 \$ de la banque à l'égard du client et non la dette du client envers la banque pour le remboursement des fonds prêtés. Par l'inscription au crédit, la banque a exécuté son obligation prévue à l'ouverture de crédit de remettre l'argent à monsieur $\mathrm{B}$. C'est cette créance de monsieur B à l'égard de la banque qui a subi l'effet novatoire par son inscription à titre de crédit au compte courant et non la créance en remboursement des fonds prêtés. Cette créance en remboursement des fonds prêtés ne subira l'effet novatoire du compte courant que lorsqu'une écriture au débit sera inscrite à cet égard. Pour les fins de l'exemple, supposons que monsieur B a 5000 \$ à son compte, c'est-à-dire que le solde du compte indique un crédit de 5000 \$à son égard. L'effet novatoire du compte courant à l'égard de la créance en remboursement des fonds prêtés n'aura lieu que lorsque la banque inscrira au débit sa créance de $1000 \$$. Elle passera l'écriture suivante : débit $1000 \${ }^{24}$ C'est à ce moment seulement que cette créance subira l'effet novatoire. Conséquemment les garanties qui pourraient être rattachées au prêt seront éteintes. ${ }^{25}$

24. Après cette écriture, le solde du compte de monsieur B indiquera donc un crédit de $4000 \$$.

25. Examinons les écritures si monsieur B est en débit pour un montant de $100 \$$ à son compte. Comme nous l'avons mentionné, l'effet novatoire du compte courant à l'égard de la créance en remboursement des fonds prêtés n'aura lieu que lorsque la banque inscrira au débit cette créance de 1000 \$. Elle passera l'écriture suivante : débit 1000 \$, ce qui signifie que le compte indiquera que le client est en débit de 1100 \$ à l'égard de la banque. Par cette inscription, cette créance subit l'effet novatoire. Les garanties qui pourraient avoir été ratta- 
Nous profitons de cette démonstration pour revenir à l'arrêt In re Hill-ADon Limited : Bank of Montréal c. Saul Kwiat (syndic) dans lequel la Cour d'appel du Québec a qualifié le solde du compte courant de prêt. ${ }^{26}$ La Cour d'appel avait mentionné que l'argent que le client dépose en banque est un prêt et que, par conséquent, le solde en compte courant est un prêt. ${ }^{27}$ Quelles sont les écritures dans la situation (que nous désignerons l'exemple numéro 2) où un client prête un montant de 2000 \$ à la banque ? La banque inscrit au compte un montant de 2000 \$ au crédit du client. Que représente cette écriture? Elle signifie que la banque a une dette de $2000 \$$ à l'égard du client. ${ }^{28}$ Contrairement au cas de l'avance décrit aux paragraphes précédents (exemple numéro 1) où la remise des fonds avait été effectuée par la banque par une écriture au crédit, la remise des fonds est effectuée ici par le client qui se rend à son institution financière remettre l'argent. Dans le cas de l'exemple numéro 2, la seule écriture au compte est celle au crédit qui matérialise la dette de la banque envers le client à l'égard des fonds prêtés : c'est la créance en remboursement des fonds prêtés qui est ainsi inscrite au compte par cette écriture, d'où l'effet novatoire subi par cette créance. Nous ne pouvons donc qualifier le solde du compte courant en fonction de l'opération ayant donné lieu à créance.

chées au prêt s'éteignent, mais cet effet extinctif n'a lieu que lorsque la banque a inscrit la créance en remboursement des fonds prêtés au débit. Mentionnons que la banque pourrait avoir un avantage à inscrire cette créance au débit même si le compte est à découvert puisque le solde du compte peut comporter des garanties.

26. Re Hill-A-Don Limited : Bank of Montréal c. Saul Kwiat (syndic), [1975] C.A. 157. Nous avons mentionné dans notre texte sur le compte courant que le solde du compte courant ne pouvait être qualifié en fonction de la créance inscrite en compte. M. Duval, supra note 1 à la p. 481 .

27. Nous favorisons dans cette situation la notion d'opération matérielle. Voir nos commentaires précédents sur ce sujet.

28. Comme mentionné précédemment, seule une écriture au crédit (dans les livres de la banque) peut matérialiser la créance en remboursement des fonds prêtés lorsque le client fait un prêt à la banque, puisque cette écriture matérialise la dette de la banque envers le client. 


\section{Le découvert en compte courant}

Un découvert en compte se produit notamment lorsqu'un client qui n'a pas suffisamment de fonds dans son compte tire un chèque pour un montant plus élevé que son solde, ceci généralement dans le cadre d'une ouverture de crédit. Ripert et Roblot mentionnent qu' « [u]ne des parties est à découvert lorsque son débit à l'égard de l'autre partie n'est pas compensé par son propre crédit. Si on clôturait le compte à ce moment-là, l'autre aurait une créance à recouvrer.» ${ }^{29} \mathrm{Il}$ sera permis au titulaire du compte d'aller en découvert lorsqu'une ouverture de crédit existe entre lui et la banque, laquelle s'oblige à faire crédit au client jusqu'à un montant déjà fixé par l'ouverture de crédit. Bien que la convention de compte courant implique une notion de crédit, «la convention des parties peut décider que le découvert sera unilatéral et aussi fixer la limite de ce découvert. ${ }^{30}$

En compte courant, en l'absence d'une ouverture de crédit qui spécifie que les sommes versées dans le cadre d'un découvert sont considérées comme étant un prêt, nous sommes d'avis que l'opération à l'origine de la créance en compte qui entraîne le découvert est une opération matérielle plutôt qu'une opération juridique. $^{31}$

Supposons pour les fins de notre exemple que la banque ait ouvert un crédit au montant de $5000 \$$ au client en compte courant (montant non encore utilisé), que les parties aient prévu que les sommes versées à cet égard constitueraient un prêt et que le client n'ait aucun fonds à son compte. Si le client

29. G. Ripert et R. Roblot, supra note 4 à la p. 313.

30. Ibid.

31. Voir les commentaires des professeurs Boistel et Escarra sur ce sujet, supra note 18. Notons également le passage suivant d'Albert Dieryck au même effet : «Puisque les versements effectués en compte par le créditeur en exécution du crédit ouvert, ne sont que des remises sans caractère spécifique qui ne participent pas à la nature de paiements ou d'avances [découvert], il [le créditeur] n'est pas définitivement libéré des obligations qu'il a assumées [dans le cadre de l'ouverture de crédit].» Il s'agit d'un des principaux avantages du compte courant, puisque «[d]ès que le crédité, qui fait des versements en compte, ne présentant pas davantage les caractères d'un paiement, a ramené le solde débiteur à un montant inférieur à celui du crédit consenti, il peut réclamer du créditeur des nouveaux versements à concurrence de la marge constituée.» A. Dieryck, supra note 3 à la p. 266. Seules des créances naissent suite aux transferts de numéraire du remettant au récepteur. 
tire un chèque au montant de $1000 \$$ sur son compte, la banque devra fournir les fonds pour l'honorer et inscrira au débit du compte le montant du chèque. Décomposons l'opération : en payant le chèque, la banque fait un prêt au montant de 1000 \$ au client, comme elle s'y était engagée par l'ouverture de crédit, pour la différence entre l'argent inscrit au compte, (zéro), et le montant du chèque. ${ }^{32}$ La créance du client envers la banque, soit l'obligation de remise de l'argent de la banque au client, a été exécutée par le paiement du chèque. Quant à la créance en remboursement des fonds prêtés de la banque envers le client, elle est inscrite au compte par l'écriture suivante : débit 1000 \$. Cette écriture matérialise la créance de la banque à l'encontre du client, d'où l'effet novatoire de cette créance en remboursement des fonds prêtés. ${ }^{33}$

32. Le chèque permet au client de retirer les fonds qu'il possède à son compte en raison de la créance qu'il détient à l'encontre de la banque ou de la caisse. Ainsi, par le chèque, le client donne un ordre à son institution financière de payer une somme précise prélevée sur les fonds qu'il possède à son compte ou à partir des fonds que va lui prêter l'institution financière en vertu d'une ouverture de crédit (en supposant que les fonds versés aient été qualifiés de prêt à l'ouverture de crédit) lorsque la somme que possède le client à son compte est insuffisante pour honorer le chèque. La nature juridique du chèque se rapproche du mandat puisque le client donne ordre à l'institution financière de prendre l'argent disponible à son compte ou, si les fonds sont insuffisants pour honorer le chèque, de prendre les fonds que cette dernière va lui prêter et de verser cette somme à son profit ou à une personne qu'il désigne.

33. Supposons que le compte du client indique un crédit de $100 \$$. Si monsieur B tire un chèque au montant de 1000 \$ sur son compte, le solde du compte sera insuffisant pour couvrir entièrement ce dernier. La banque devra donc fournir les fonds pour l'honorer en inscrivant au débit du compte le montant constatant la différence entre l'argent inscrit au crédit du compte et le montant du chèque tiré sur celui-ci. Décomposons l'opération : en payant le chèque, la banque exécute, dans un premier temps, la créance du client à son égard, à savoir le paiement du montant de 100 \$ que possède le client à son compte. Rappelons que ce montant de 100 \$ au crédit du client, dans les livres de la banque, représente une créance exigible en tout temps du client à l'égard de la banque. Dans un deuxième temps, la banque fait un prêt au montant de 900 \$ au client, pour la différence entre l'argent inscrit au compte, c'est à dire le $100 \$$ au crédit du client, et le montant du chèque. La créance du client envers la banque relativement au prêt de 900 \$, soit l'obligation de la banque de remettre l'argent au client en vertu de l'obligation prévue à l'ouverture de crédit, a été exécutée par le paiement du chèque par la banque. L'écriture inscrite au compte par la banque est la suivante : débit 1000 \$. Du montant de 1000 \$ inscrit au débit, un montant de 100 \$ matérialise l'exécution de la créance du client envers la banque à l'égard du solde du compte et un montant de $900 \$$ matérialise la créance de la banque en remboursement des fonds prêtés. Le solde du compte indiquera un montant de 900 \$ au débit. 
Le lecteur peut trouver surprenant que la créance en remboursement des fonds prêtés subisse l'effet novatoire du compte courant et qu'en conséquence le prêt n'ait subsisté que quelques instants. Nous soulignons que cette situation n'est pas différente de celle qui se produit dans le cas d'une créance de prix de vente qui est inscrite en compte courant entre un entrepreneur en construction et un marchand de matériaux. Supposons qu'une ouverture de crédit est accordée à l'entrepreneur par le marchand de matériaux pour un montant de 5000 \$. Pour les fins de notre exemple, assumons qu'une vente est conclue et que l'entrepreneur achète pour $1000 \$$ de matériaux. L'entrepreneur a l'obligation de payer le prix en contrepartie de la chose qu'il reçoit. La créance du prix de vente est inscrite immédiatement après que la vente ait été conclue puisqu'elle est un contrat consensuel. ${ }^{34}$ L'écriture relative à cette créance se traduira par l'inscription suivante : débit 1000 \$. Par l'inscription de cette créance au compte, celle-ci subit l'effet novatoire.

\section{L'effet novatoire et les ouvertures de crédit au Québec}

Le découvert et la protection contre le découvert sont les deux types de clause que nous retrouvons le plus fréquemment dans les conventions d'ouverture de crédit.

\section{1) Le découvert}

Voici une clause de découvert jointe à un compte courant que nous retrouvons dans une convention d'ouverture de crédit :

«Marge de crédit par découvert. J'utiliserai la marge de crédit par découvert uniquement pour les besoins de liquidités d'exploitation de mon entreprise. Ma limite de crédit s'applique seulement au numéro de compte d'affaires indiqué à la première page de la présente convention (mais, si le crédit $\mathrm{E}$ est aussi coché, voir la clause 9 ci-après). Je suis responsable de tous les débits que j'ai amorcés (tels les chèques, les paiements au titre d'un prêt, les prélèvements automatiques, etc.). Les

34. Vasseur et Marin mentionnent que «[s]i la marchandise est de celles qui ne réclament aucun agrément de l'acheteur et si la vente est définitive au jour du contrat, le prix doit passer au compte au jour de la vente.» M. Vasseur et X. Marin, supra note 16 à la p. 400. 
paiements se font au moyen de dépôts dans le compte. Je ne devrai jamais dépasser la limite de crédit. Vous, la CIBC, pouvez sans m'aviser retourner tout débit porté au compte dont le paiement entraînerait le dépassement de la limite de crédit, sauf entente préalable entre nous. Si vous payez un tel débit, je vous rembourserai immédiatement l'excédent sur la limite de crédit.» ${ }^{35}$

Cette clause permet au titulaire du compte courant d'aller à découvert jusqu'à concurrence du montant accordé par son institution financière. Le compte est à découvert lorsque les fonds sont insuffisants notamment pour honorer un chèque. Ainsi, si le titulaire du compte tire un chèque au montant de $500 \$$ alors qu'il ne possède aucun fonds à son compte, le compte sera à découvert. La banque matérialisera le transfert de fonds par l'écriture suivante : débit 500 \$. Il s'agit d'une opération matérielle; seules des créances naissent pour matérialiser le transfert de numéraire de la banque au titulaire du compte dans le cas du découvert et du titulaire du compte à la banque lorsque le titulaire diminue son solde débiteur, le ramène à zéro ou le rend positif.

Si le découvert a été qualifié de prêt, l'opération à l'origine du transfert de fonds aurait alors constitué un prêt au montant de $500 \$ .^{36}$ La créance en remboursement des fonds prêtés aurait été inscrite au compte par l'écriture suivante : débit 500 \$. Par cette inscription, cette créance aurait été remplacée par le solde du compte, d'où la disparition du prêt. D'autre part, si ce prêt fait par la

35. Soulignons que cette clause parle de paiement au compte courant, alors que cette notion ne reflète pas la théorie du compte courant. Voir à ce sujet M. Duval, supra note 1 à la p. 467. Banque Canadienne Impériale de Commerce. Voici un autre exemple d'une clause de découvert tirée de la Convention de découvert de la Banque Royale du Canada :

«Utilisation du compte : Je peux obtenir des avances de fonds en effectuant des tirages sur le compte. Vous imputez le montant que vous m'avancez comme un tirage sur le compte. Ce montant fera partie du découvert à compter du jour où vous l'aurez porté au compte.

[...]

Le compte constitue une marge de crédit renouvelable. À chaque utilisation, le découvert créé est soustrait du plafond autorisé. À chaque versement (qui réduit le découvert que je vous dois), le plafond est reconstitué d'autant.»

36. Un exemple de la qualification à titre de prêt est la Convention de Multicompte de la Banque de Montréal qui définit à la clause 1.02 le terme Prêt comme étant «le découvert du compte, c'est-à-dire le total des articles portés au débit du compte, notamment, sans limiter la généralité de ce qui précède, les chèques, et les frais qui excèdent le total des articles portés au crédit du compte.» 
banque avait été garanti par une hypothèque, celle-ci aurait disparu en même temps que le prêt par l'inscription au compte de la créance en remboursement des fonds prêtés.

\section{2) La protection contre le découvert : l'avance de fonds}

Un autre type de clause est la protection contre le découvert. Ainsi, dès que le compte devient à découvert, il est approvisionné afin d'éviter qu'il demeure à découvert. Une telle clause se lit comme suit :

«Protection contre découvert : Sauf dans le cas d'une marge de crédit rénovation, le Client autorise la Banque à utiliser la Marge de crédit pour prévenir tout découvert au compte qu'il désigne à cette fin. La Banque pourra débiter le Compte pour un montant suffisant à cette fin et effectuer les virements de fonds nécessaires. La Banque n'est toutefois pas tenue d'utiliser la Marge de crédit à cette fin si l'utilisation devait entraîner un dépassement de la Limite de crédit. Le Client peut révoquer l'autorisation ci-dessus au moyen d'un préavis de cinq jours adressé à la Banque à la succursale où le Compte est tenu. La Banque peut en tout temps refuser d'agir conformément à cette autorisation en donnant un avis au Client à cet effet. » ${ }^{37}$

37. Convention de marge de crédit de la Banque Nationale du Canada. Certains pourraient arguer que, puisqu'il s'agit d'une protection contre découvert, le compte visé par cette protection ne peut être un compte courant.

Afin de déterminer si nous sommes en présence d'un compte courant, il faut notamment qu'il y ait possibilité de remises réciproques même si elles n'ont pas lieu (voir nos commentaires sur les éléments qui permettent de déterminer si nous sommes en présence d'un compte courant à notre article sur le sujet. M. Duval, supra note 1.). Or, nous constatons par les écritures comptables, que le compte permet d'aller à découvert bien qu'il ne demeure pas dans cette position (à l'égard du client de la banque). D'autre part, Madame Nicole L'Heureux est d'avis que le « compte bancaire avec privilège de chèques fonctionne selon les principes du compte courant»; N. L'Heureux, Droit bancaire, $3^{\mathrm{e}}$ éd., Cowansville (Qc.), Yvon Blais, 1999 à la p. 72. En outre, même le compte de dépôt fonctionne comme un compte courant : Marie-Thérèse Rives-Lange mentionne que : «les deux comptes mettent en jeu le même mode de règlement, l'effet novatoire : en tant que mode de règlement des créances, le compte de dépôt est donc assimilable au compte courant. Mais il existe dans le compte courant une convention d'affectation générale absente dans le compte de dépôt : en tant que règle d'origine conventionnelle le compte courant se distingue du compte de dépôt.» M.-T. Rives-Lange, supra note 10 à la p. 68. 
Qu'arrive-t-il dans cette situation? La banque approvisionne le compte courant afin qu'il ne demeure pas à découvert. Supposons que monsieur A, détenteur d'une ouverture de crédit avec la clause ci-dessus, possède un compte à la banque $\mathrm{X}$ et qu'il n'ait aucun fonds à ce dernier (supposons pour les fins de notre exemple que l'opération à l'origine de la créance inscrite en compte qui crée le découvert constitue une opération matérielle plutôt qu'un prêt). ${ }^{38}$ Si monsieur A tire un chèque au montant de $1000 \$$, la banque inscrira un montant de $1000 \$$ au débit du compte. Afin d'assumer son obligation prévue à l'ouverture de crédit qui prévoit la protection contre le découvert, la banque fait un prêt de 1000 à monsieur A immédiatement après avoir inscrit au débit du compte le montant de $1000 \$$ mentionné plus haut. À cet égard, une écriture de $1000 \$$ au crédit du compte est effectuée par la banque. Comment expliquer les deux écritures soit une au débit et une au crédit? L'écriture au débit représente la créance de la banque à l'égard de monsieur A pour le transfert de fonds afin d'honorer le chèque. En effet, la banque en acceptant l'ordre de paiement du chèque alors que le solde du compte indiquait zéro, a déboursé un montant de 1000 \$. L'écriture au débit de 1000 \$ représente la créance de la banque envers monsieur A à la suite du transfert de fonds : il s'agit d'un découvert.

Immédiatement après l'écriture au débit, la banque en inscrit une autre, celle de 1000 \$ au crédit du compte qui est la protection contre le découvert : il s'agit d'une avance de fonds, un prêt. Après ces écritures, le solde du compte indiquera le chiffre zéro. Nous constatons que le montant inscrit au crédit représente la créance résultant de l'obligation afférente à l'ouverture de crédit. La banque avait l'obligation d'avancer des fonds afin de protéger monsieur $\mathrm{A} d u$ découvert bancaire. ${ }^{39}$ Elle s'est acquittée de son obligation envers monsieur A par l'inscription au crédit du compte. Ce n'est pas la créance en remboursement des fonds prêtés résultant de l'avance qui a été inscrite au compte, mais celle qui résulte de l'obligation prévue à l'ouverture de crédit qui a été exécutée par l'inscription au compte. Le prêt existe donc toujours avec les sûretés qui pourraient avoir été accordées par monsieur A afin de garantir le prêt résultant de l'avance de la banque.

38. À supposer que ce découvert soit qualifié de prêt, ce serait la créance en remboursement des fonds prêtés qui serait inscrite au compte, d'où la disparition du prêt.

39. Obligation implicite sous réserve de certaines conditions. 


\section{Conclusion}

L'analyse de l'avance et du découvert en compte courant nous a permis de constater l'importance pour le praticien d'examiner attentivement la créance entrée en compte afin de déterminer à quel moment les sûretés qui peuvent garantir une créance sont éteintes. Ainsi, nous avons établi dans le cadre de l'avance en compte courant que les sûretés afférentes à celle-ci demeurent lors de l'inscription au crédit et disparaissent au moment où la banque inscrit au débit du compte la créance en remboursement des fonds prêtés. Lors d'un découvert bancaire en compte courant où l'opération a été qualifiée de prêt, les garanties afférentes au prêt disparaissent immédiatement puisqu'il y a immédiatement inscription au débit du compte de la créance en remboursement des fonds prêtés après la remise de l'argent du prêteur à l'emprunteur. Il y a donc lieu, dans le cas du découvert bancaire, que les institutions financières garantissent le solde du compte courant plutôt que le prêt puisque la créance en remboursement des fonds prêtés, par l'inscription au compte et par l'effet novatoire qui découle de cette inscription, est remplacée par le solde du compte courant. 